\title{
Linked Partitions and Permutation Tableaux
}

\author{
William Y.C. Chen \\ Center for Applied Mathematics \\ Tianjin University \\ Tianjin 300072, P.R. China \\ chenyc@tju.edu.cn \\ Lewis H. Liu \\ Center for Combinatorics, LPMC-TJKLC \\ Nankai University \\ Tianjin 300071, P.R. China \\ lewis@cfc.nankai.edu.cn \\ Carol J. Wang \\ Department of Mathematics \\ Beijing Technology and Business University \\ Beijing 100048, P.R. China \\ wang_jian@th.btbu.edu.cn
}

Submitted: May 23, 2013; Accepted: Sep 19, 2013; Published: Sep 26, 2013

Mathematics Subject Classifications: 05A05, 05A19

\begin{abstract}
Linked partitions were introduced by Dykema in the study of transforms in free probability theory, whereas permutation tableaux were introduced by Steingrímsson and Williams in the study of totally positive Grassmannian cells. Let $[n]=\{1,2, \ldots, n\}$. Let $L(n, k)$ denote the set of linked partitions of $[n]$ with $k$ blocks, let $P(n, k)$ denote the set of permutations of $[n]$ with $k$ descents, and let $T(n, k)$ denote the set of permutation tableaux of length $n$ with $k$ rows. Steingrímsson and Williams found a bijection between the set of permutation tableaux of length $n$ with $k$ rows and the set of permutations of $[n]$ with $k$ weak excedances. Corteel and Nadeau gave a bijection between the set of permutation tableaux of length $n$ with $k$ columns and the set of permutations of $[n]$ with $k$ descents. In this paper, we establish a bijection between $L(n, k)$ and $P(n, k-1)$ and a bijection between $L(n, k)$ and $T(n, k)$. Restricting the latter bijection to noncrossing linked partitions and nonnesting linked partitions, we find that the corresponding permutation tableaux can be characterized by pattern avoidance.
\end{abstract}

Keywords: linked partition; permutation; descent; permutation tableau. 


\section{Introduction}

The notion of linked partitions was introduced by Dykema [8] in the study of the unsymmetrized T-transform in free probability theory. Let $[n]=\{1,2, \ldots, n\}$. A linked partition of $[n]$ is a collection of nonempty subsets $B_{1}, B_{2}, \ldots, B_{k}$ of $[n]$, called blocks, such that the union of $B_{1}, B_{2}, \ldots, B_{k}$ is $[n]$ and any two distinct blocks are nearly disjoint. Two blocks $B_{i}$ and $B_{j}$ are said to be nearly disjoint if for any $k \in B_{i} \cap B_{j}$, one of the following conditions holds:

(a) $k=\min \left(B_{i}\right),\left|B_{i}\right|>1$ and $k \neq \min \left(B_{j}\right)$, or

(b) $k=\min \left(B_{j}\right),\left|B_{j}\right|>1$ and $k \neq \min \left(B_{i}\right)$.

The linear representation of a linked partition was introduced by Chen, $\mathrm{Wu}$ and Yan [1]. For a linked partition $\tau$ of $[n]$, list the $n$ vertices in a horizontal line with labels $1,2, \ldots, n$. For a block $B=\left\{i_{1}, i_{2}, \ldots, i_{k}\right\}$ with $k \geqslant 2$ and $i_{1}<i_{2}<\cdots<i_{k}$, draw an arc from $i_{1}$ to $i_{j}$ for $j=2, \ldots, k$. For example, the linear representation of the linked partition $\{1,2,4\}\{2,3\}\{3,9\}\{5,6\}\{6,7\}\{8\}$ is illustrated in Figure 1.1.

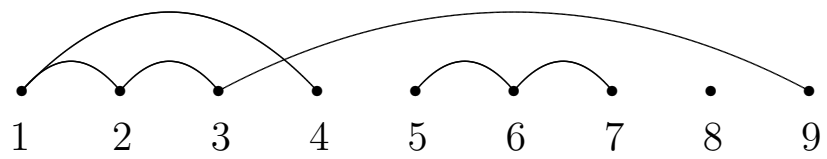

Figure 1.1: The linear representation of a linked partition.

For $i<j$, we use a pair $(i, j)$ to denote an arc from $i$ to $j$, and we call $i$ and $j$ the lefthand endpoint and the right-hand endpoint of $(i, j)$, respectively. Two arcs $\left(i_{1}, j_{1}\right)$ and $\left(i_{2}, j_{2}\right)$ form a crossing if $i_{1}<i_{2}<j_{1}<j_{2}$, and form a nesting if $i_{1}<i_{2}<j_{2}<j_{1}$. For the linked partition in Figure 1.1, there is one crossing formed by $(1,4)$ and $(3,9)$, while there are three nestings: $(1,4)$ and $(2,3),(3,9)$ and $(5,6),(3,9)$ and $(6,7)$. A linked partition is called noncrossing (resp., nonnesting) if there does not exist any crossing (resp., nesting) in its linear representation. Dykema [8] showed that the number of noncrossing linked partitions of $[n+1]$ is equal to the $n$-th large Schröder number. The sequence of the large Schröder numbers is listed as A006318 in OEIS [9]. Chen, Wu and Yan [1] found a bijective proof of this fact and proved that the number of nonnesting linked partitions of $[n]$ is also equal to the number of noncrossing linked partitions of $[n]$.

Permutation tableaux were introduced by Steingrímsson and Williams [11] in the study of totally positive Grassmannian cells. They are closely related to the PASEP (partially asymmetric exclusion process) model in statistical physics $[2,4,5,6]$. Permutation tableaux are also in one-to-one correspondence with alternative tableaux introduced by Viennot [12]. More precisely, a permutation tableau is defined as a Ferrers diagram possibly with empty rows such that the cells are filled with 0's and 1's, and

(1) each column contains at least one 1 ;

(2) there does not exist any 0 with a 1 above (in the same column) and a 1 to the left (in the same row). 
The length of a permutation tableau is defined as the number of rows plus the number of columns. A permutation tableau $T$ of length $n$ is labeled by the elements in $[n]$ in increasing order from the top right corner to the bottom left corner, see Figure 1.2. We use $(i, j)$ to denote the cell in row $i$ and column $j$. The shape of a permutation tableau $T$ is meant to be the shape of the underlying Ferrers diagram of $T$ with empty rows allowed. In other words, the shape of $T$ is a partition $\left(\lambda_{1}, \lambda_{2}, \ldots, \lambda_{k}\right)$, where $\lambda_{i}$ is the number of cells in $i$-th row of the underlying Ferrers diagram of $T$, for $i=1,2, \ldots, k$. For example, Figure 1.2 gives a permutation tableau of shape $(5,5,3,2,1,0)$ and length 11 .

\begin{tabular}{|c|c|c|c|c|}
\hline 1 & 0 & 0 & 1 & 0 \\
\hline 0 & 1 & 0 & 1 & 1 \\
\hline 0 & 0 & 1 & $5^{4}$ & 3 \\
\hline 0 & 0 & 6 & & \\
\hline & $9^{8}$ & & & \\
\hline
\end{tabular}

Figure 1.2: A permutation tableau of length 11 with shape $(5,5,3,2,1,0)$.

Corteel and Nadeau [3] found a bijection from permutation tableaux of length $n$ with $k$ columns and permutations of $[n]$ with $k$ descents. Steingrímsson and Williams [11] established a one-to-one correspondence between permutation tableaux of length $n$ with $k$ rows and permutations of $[n]$ with $k$ weak excedances.

This paper aims to demonstrate that linked partitions play a role as an intermediate structure between permutations and permutation tableaux. To this end, we present a bijection between linked partitions and permutations, and a bijection between linked partitions and permutation tableaux. In fact, the first bijection maps a linked partition of $[n]$ with $k$ blocks to a permutation of $[n]$ with $k-1$ descents, and the second bijection transforms a linked partition of $[n]$ with $k$ blocks to a permutation tableau of length $n$ with $k$ rows.

Combining the above two bijections, we are led to a one-to-one correspondence between permutations and permutation tableaux, which implies some known properties of permutation tableaux obtained by Corteel and Nadeau [3] and Steingrímsson and Williams [11].

When restricting the second bijection to noncrossing linked partitions, we find that the corresponding permutation tableaux are exactly those that avoid a pattern $J_{2}$. Similarly, when restricting this bijection to nonnesting linked partitions, we get permutation tableaux that avoid a pattern $I_{2}$. The definitions of the patterns $I_{2}$ and $J_{2}$ are given in Section 4. 


\section{Linked partitions and permutations}

In this section, we present a bijection between linked partitions of $[n]$ with $k$ blocks and permutations of $[n]$ with $k-1$ descents.

To describe the construction of this bijection, we give a classification of vertices in the linear representation of a linked partition. Let $\tau$ be a linked partition of $[n]$. A vertex $i$ in the linear representation of $\tau$ is called an origin if it is only a left-hand endpoint, or a transient if it is both a left-hand point and a right-hand endpoint, or a singleton if it is an isolated vertex, or a destination if it is only a right-hand endpoint. Figure 2.1 illustrates the four types of vertices.

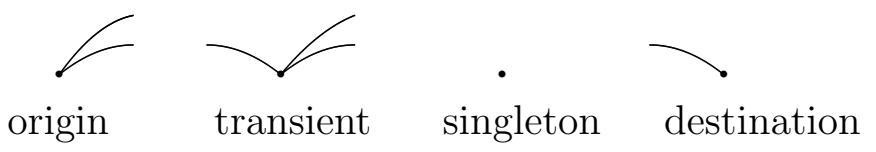

Figure 2.1: Four types of vertices in linked partitions.

Let $L(n, k)$ denote the set of linked partitions of $[n]$ with $k$ blocks. Let $\pi=\pi_{1} \pi_{2} \cdots \pi_{n}$ be a permutation of $[n]$. An integer $i(1 \leqslant i \leqslant n-1)$ is called a decent (resp., ascent) of $\pi$ if $\pi_{i}>\pi_{i+1}$ (resp., $\pi_{i}<\pi_{i+1}$ ). Let $P(n, k)$ denote the set of permutations of $[n]$ with $k$ descents, which is counted by the Eulerian number $A(n, k+1)$, see, for example, Stanley $[10]$.

Theorem 2.1. For $n \geqslant 1$ and $1 \leqslant k \leqslant n$, there is a bijection $\varphi$ between $L(n, k)$ and $P(n, k-1)$.

Proof. We conduct induction on $n$. If $n=1$, that is, $\tau=\{1\}$. In this case, we have $k=1$. Then we set $\varphi(\tau)=1$, which is a permutation with no descent. So the theorem holds for $n=1$.

We now assume that $n \geqslant 2$ and that the theorem holds for $n-1$. We aim to construct a map $\varphi$ from $L(n, k)$ to $P(n, k-1)$, which is easily seen to be a bijection. To define $\varphi$, let $\tau \in L(n, k)$ be a linked partition of $[n]$ with $k$ blocks and let $\tau^{\prime}$ denote the linked partition of $[n-1]$ obtained from $\tau$ by removing the vertex $n$ along with the arcs possibly associated to $n$. Starting with $\varphi\left(\tau^{\prime}\right)$, we proceed to generate a permutation $\varphi(\tau)$ of $[n]$ with $k-1$ descents.

Suppose that $\tau^{\prime}$ contains $s$ blocks. Notice that $n$ is either a singleton or a destination in $\tau$. If $n$ is a singleton, then it is clear that $s=k-1$; if $n$ is a destination, then we have $s=k$. By the induction hypothesis, $\varphi\left(\tau^{\prime}\right)$ is a permutation of $[n-1]$ with $s-1$ descents. Now $\varphi(\tau)$ can be defined by inserting $n$ into the permutation $\varphi\left(\tau^{\prime}\right)$. More precisely, let $\varphi\left(\tau^{\prime}\right)=\pi_{1} \pi_{2} \cdots \pi_{n-1}$. Let $i_{1}, i_{2}, \ldots, i_{s}$ be the minimum elements of blocks in $\tau^{\prime}$ listed in increasing order, and let $j_{1}, j_{2}, \ldots, j_{t}$ be the destinations of $\tau^{\prime}$ listed also in increasing order. Clearly, we have $t=n-1-s$. Assume that $m$ is the minimum element in the block of $\tau$ that contains $n$. To ensure that $\varphi$ is a bijection, we need to find a position to insert $n$ into $\varphi\left(\tau^{\prime}\right)$ such that the inserting procedure is reversible. There are four cases. 
Case 1: $m=i_{r}$, where $1 \leqslant r \leqslant s-1$, that is, there is an arc $\left(i_{r}, n\right)$ in the linear representation of $\tau$. Set

$$
\varphi(\tau)=\pi_{1} \pi_{2} \cdots \pi_{\ell} n \pi_{\ell+1} \cdots \pi_{n-1},
$$

where $\ell$ is the $r$-th descent of $\varphi\left(\tau^{\prime}\right)$ from left to right.

Case 2: $m=i_{s}$. Set

$$
\varphi(\tau)=\varphi\left(\tau^{\prime}\right) n
$$

Case 3: $m=j_{r}$, where $1 \leqslant r \leqslant t$. Set

$$
\varphi(\tau)=\pi_{1} \pi_{2} \cdots \pi_{\ell} n \pi_{\ell+1} \cdots \pi_{n-1},
$$

where $\ell$ is the $r$-th ascent in $\varphi\left(\tau^{\prime}\right)$ from left to right.

Case 4: $m=n$, that is, $n$ is a singleton in $\tau$. Set

$$
\varphi(\tau)=n \varphi\left(\tau^{\prime}\right)
$$

Clearly, $\varphi(\tau)$ is a permutation of $[n]$ in any of the above cases. It remains to prove that $\varphi(\tau)$ has $k-1$ descents. Here we shall only give the proof for Case 1 , since the other cases can be justified by the same reasoning. In Case 1 , there is an arc $(m, n)$ in $\tau$, where $m$ is the minimum element of a block of $\tau$. Hence $\tau^{\prime}$ has the same number of blocks as $\tau$, namely, $\tau^{\prime}$ belongs to $L(n-1, k)$. By the induction hypothesis, $\varphi\left(\tau^{\prime}\right)$ belongs to $P(n-1, k-1)$. It is clear from $(2.1)$ that $\varphi(\tau)$ has the same number of descents as $\varphi\left(\tau^{\prime}\right)$. Thus $\varphi(\tau)$ has $k-1$ descents.

It is not difficult to see that the above procedure is reversible. Hence the map $\varphi$ is a

\begin{tabular}{|c|c|c|c|c|c|}
\hline$\{1,2,3\}$ & $\{1,2\}\{2,3\}$ & $\{1,3\}\{2\}$ & $\{1,2\}\{3\}$ & $\{1\}\{2,3\}$ & $\{1\}\{2\}\{3\}$ \\
\hline & & & & & $i$ \\
\hline 123 & 132 & 231 & 312 & 213 & 321 \\
\hline
\end{tabular}
bijection, and the proof is complete by induction.

For example, Figure 2.2 illustrates the linked partitions of $\{1,2,3\}$ and the corresponding permutations.

Figure 2.2: Linked partitions of $\{1,2,3\}$ and the corresponding permutations.

\section{Linked partitions and permutation tableaux}

The objective of this section is to present a bijection between linked partitions of $[n]$ with $k$ blocks and permutation tableaux of length $n$ with $k$ rows. As consequences, we obtain equidistribution properties between linked partitions and permutation tableaux 
with certain restrictions. For example, we find that the number of permutation tableaux of length $n$ with $k$ rightmost restricted 0's is equal to the number of linked partitions of $[n]$ with $k$ transients. Our bijections also lead to some known results on permutation tableaux obtained by Corteel and Nadeau [3] and Steingrímsson and Williams [11].

To construct the bijection between linked partitions and permutation tableaux, we introduce the shape of a linked partition. Let $\tau$ be a linked partition of $[n]$. Using the linear representation of $\tau$, we obtain a sequence $S=s_{1} s_{2} \cdots s_{n}$ by letting $s_{i}=H$ if $i$ is a destination, and $s_{i}=V$ otherwise, where $H$ stands for the horizontal step $(-1,0)$ and $V$ stands for the vertical step $(0,-1)$. Consider the sequence $S$ as a lattice path, and let $\lambda$ be the Ferrers diagram of a partition having the lattice path $S$ as its boundary from the top right corner to the bottom left corner. This partition $\lambda$ with empty parts allowed is defined as the shape of the linked partition $\tau$. For example, Figure 3.1 demonstrates the shape of the linked partition in Figure 1.1.

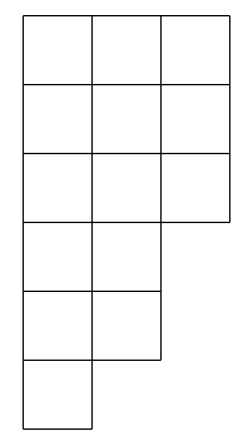

Figure 3.1: The shape of linked partition $\{1,2,4\}\{2,3\}\{3,9\}\{5,6\}\{6,7\}\{8\}$.

The construction of our bijection also requires a fact proved by Corteel and Nadeau [3] that a permutation tableau is determined by its topmost 1's and rightmost restricted 0's. By a topmost 1 we mean the topmost 1 in a column. A 0 is said to be restricted if it is below a 1 in the same column. A rightmost restricted 0 means the rightmost restricted 0 in a row. For example, in the permutation tableau in Figure 1.2, the topmost 1's are in the cells $(1,4),(1,10),(2,3),(2,8)$ and $(5,6)$, while the rightmost restricted 0 's are in the cells $(2,10),(5,8)$ and $(7,8)$. To see that a permutation tableau is uniquely determined by its topmost 1's and rightmost restricted 0's, it suffices to observe the fact that if the positions of topmost 1's are given, then all the cells above these positions (in the same columns) are filled with 0's; if the positions of the rightmost restricted 0's are given, then all the cells to the left of these positions (in the same rows) are filled with 0's; and the remaining cells are filled with 1 's.

Let $T(n, k)$ denote the set of permutation tableaux of length $n$ with $k$ rows. We have the following correspondence and the explicit construction is given in the proof.

Theorem 3.1. For $n \geqslant 1$ and $1 \leqslant k \leqslant n$, there is a shape preserving bijection $\phi$ between $L(n, k)$ and $T(n, k)$. 
Proof. Let $\tau$ be a linked partition in $L(n, k)$. We construct a permutation tableau $T=$ $\phi(\tau)$ of the same shape as $\tau$.

First, we generate the shape $\lambda$ of $\tau$, and label the boundary of $\lambda$ by using the elements of $[n]$ in increasing order from the top right corner to the bottom left corner.

Next, we fill the cells of the diagram of $\lambda$ with topmost 1's and rightmost restricted 0 's. Let $i_{1}$ be the minimum origin in $\tau$, and let $j$ be the largest destination such that there exists a path $\left(i_{1}, i_{2}, \ldots, i_{m}, j\right)$ from $i_{1}$ to $j$ in the linear representation of $\tau$. Then we fill the cell $\left(i_{1}, j\right)$ with 1 . For $\ell=2, \ldots, m$, we fill the cells $\left(i_{\ell}, j\right)$ with 0 .

Let $\tau^{\prime}$ be the linked partition of $[n]$ obtained from $\tau$ by removing the arcs in the path $\left(i_{1}, i_{2}, \ldots, i_{m}, j\right)$. Repeating the above process for $\tau^{\prime}$, we can fill each column with a 1 possibly along with some 0's until there are no arcs left in the linear representation of $\tau$.

Finally, we obtain a permutation tableau $T$ for which the 1's and 0's filled in $\lambda$ are the topmost 1's and rightmost restricted 0's of $T$, respectively.

The inverse map of $\phi$ can be described as follows. Let $T$ be a permutation tableau of length $n$ and shape $\lambda$. We construct a linked partition $\tau$ of $[n]$ such that $T=$ $\phi(\tau)$. For the column labeled with $j$, let $\left(i_{1}, j\right)$ be the cell filled with a topmost 1 and $\left(i_{2}, j\right),\left(i_{3}, j\right), \ldots,\left(i_{m}, j\right)$ be the cells filled with the rightmost restricted 0 's. For $\ell=1,2, \ldots, m$, let $\left(i_{\ell}, i_{\ell+1}\right)$ be an arc in the linear representation of $\tau$, where $i_{m+1}=j$. Note that there is a unique topmost 1 in each column and at most one rightmost restricted 0 in each row. Thus, for any vertex $j$ in $\tau$, there is at most one arc whose right-hand endpoint is $j$. This implies that $\tau$ is a linked partition of $[n]$. Moreover, it is not difficult to see that $T$ and $\tau$ have the same shape. This completes the proof.

For example, the permutation tableau corresponding to the linked partition in Figure 1.1 is given in Figure 3.2.

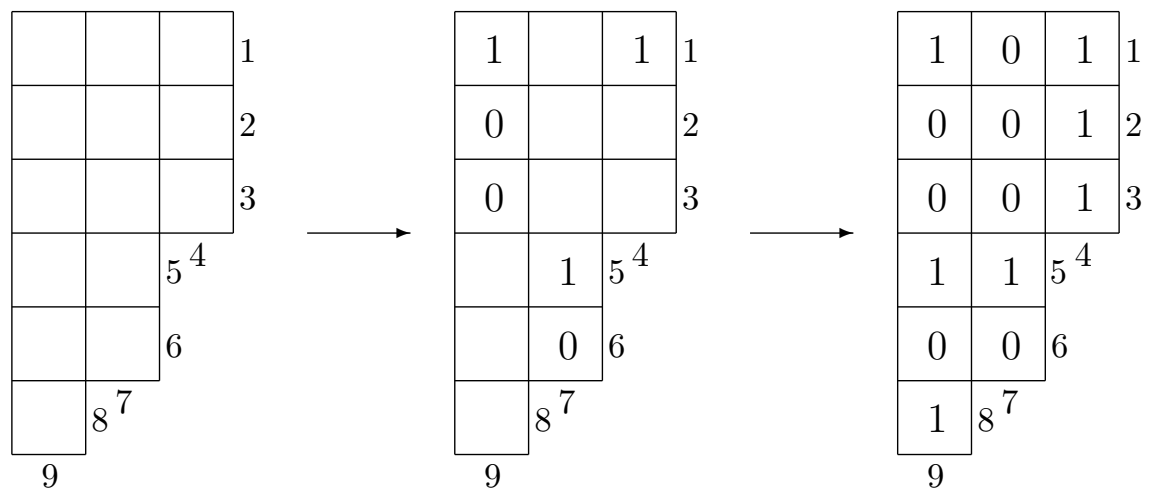

Figure 3.2: From a linked partition to a permutation tableau.

For the permutation tableau given in Figure 1.2, the corresponding linked partition is given in Figure 3.3.

The bijection $\phi$ has the following properties. 


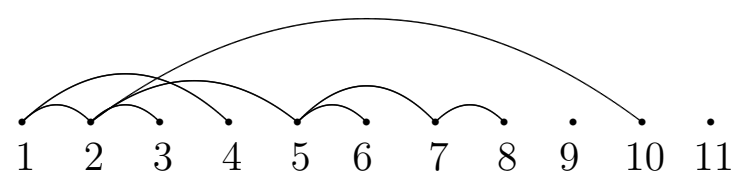

Figure 3.3: The linked partition corresponding to the permutation tableau in Figure 1.2.

Corollary 3.2. For $n \geqslant 1$, let $\tau$ be a linked partition of $[n]$, and let $T=\phi(\tau)$. Then the number of arcs in the linear representation of $\tau$ is equal to the total number of topmost 1 's and rightmost restricted 0 's in $T$.

Corollary 3.3. Assume that $n \geqslant 2$.

(1) For $0 \leqslant k \leqslant n-2$, the number of linked partitions of $[n]$ with $k$ transients equals the number of permutation tableaux of length $n$ with $k$ rightmost restricted 0 's;

(2) For $0 \leqslant k \leqslant n$, the number of linked partitions of $[n]$ with $k$ singletons equals the number of permutation tableaux of length $n$ in which there are $k$ rows that do not contain any topmost 1 or restricted 0 .

To conclude this section, we remark that the bijections $\varphi$ and $\phi$ lead to some known results on permutation tableaux. Recall that $P(n, k)$ denotes the set of permutations of $[n]$ with $k$ descents and $T(n, k)$ denotes the set of permutation tableaux of length $n$ with $k$ rows. Let $P^{\prime}(n, k)$ be the set of permutations of $[n]$ with $k$ excedances, and $T^{\prime}(n, k)$ be the set of permutation tableaux of length $n$ with $k$ columns. On one hand, since $|P(n, k-1)|=|P(n, n-k)|$, we see that $|T(n, k)|=|P(n, n-k)|$, which is equivalent to the relation $\left|T^{\prime}(n, k)\right|=|P(n, k)|$ given by Corteel and Nadeau [3]. On the other hand, it is well-known that $|P(n, k-1)|=\left|P^{\prime}(n, k)\right|$, see, for example, Stanley [10, Chapter 1]. This implies the relation $|T(n, k)|=\left|P^{\prime}(n, k)\right|$ proved by Steingrímsson and Williams $[11]$.

\section{Pattern avoiding permutation tableaux}

In this section, we discuss restrictions of the bijection $\phi$ in Section 3 to noncrossing linked partitions and nonnesting linked partitions, and we characterize the corresponding permutation tableaux by pattern avoidance.

We introduce two patterns $I_{2}$ and $J_{2}$ as given in Figure 4.1, where a dot means a topmost 1 or a rightmost restricted 0 in a permutation tableau. We choose $I_{2}$ and $J_{2}$ to denote these two patterns because $I_{t}$ and $J_{t}$ are used to stand for a diagonal chain of length $t$ and an antidiagonal chain of length $t$ in the context of fillings of Ferrers diagrams, see de Mier [7].

A permutation tableau that avoids the pattern $I_{2}$ or the pattern $J_{2}$ can be defined as follows. Let $T$ be a permutation tableau, and let $T^{\prime}$ be the permutation tableau obtained from $T$ by replacing the topmost 1's and rightmost restricted 0's by dots and removing 

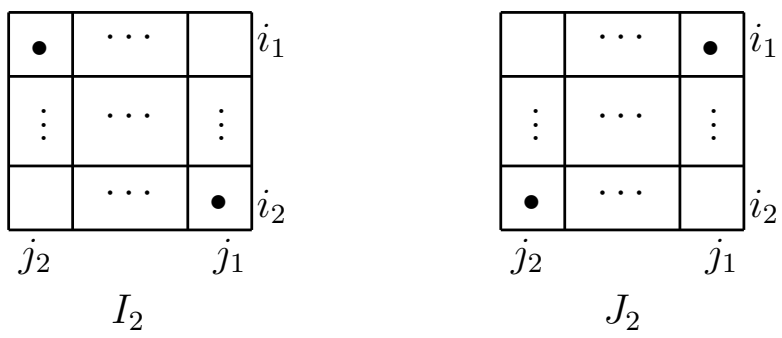

Figure 4.1: Patterns $I_{2}$ and $J_{2}$.

all other 1's and 0's. We say that $T$ avoids the pattern $I_{2}$ if $T^{\prime}$ does not contain four cells $\left(i_{1}, j_{1}\right),\left(i_{1}, j_{2}\right),\left(i_{2}, j_{1}\right)$ and $\left(i_{2}, j_{2}\right)$, where $i_{1}<i_{2}<j_{1}<j_{2}$, such that the cells $\left(i_{1}, j_{2}\right)$ and $\left(i_{2}, j_{1}\right)$ are filled with dots, while the cell $\left(i_{2}, j_{2}\right)$ is empty. Similarly, we say that $T$ avoids the pattern $J_{2}$ if $T^{\prime}$ does not contain four cells $\left(i_{1}, j_{1}\right),\left(i_{1}, j_{2}\right),\left(i_{2}, j_{1}\right)$ and $\left(i_{2}, j_{2}\right)$, where $i_{1}<i_{2}<j_{1}<j_{2}$, such that the cells $\left(i_{1}, j_{1}\right)$ and $\left(i_{2}, j_{2}\right)$ are filled with dots, while the cell $\left(i_{2}, j_{1}\right)$ is empty. For example, Figure 4.2 gives a permutation tableau avoiding the pattern $I_{2}$. Note that this permutation tableau contains the pattern $J_{2}$, as exemplified by the cells $\{(1,3),(1,6),(2,3),(2,6)\}$.

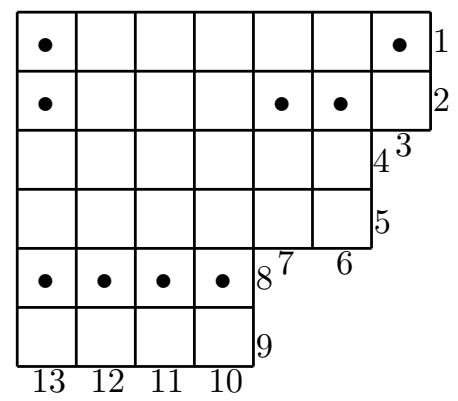

Figure 4.2: A permutation tableau avoiding $I_{2}$.

By the construction of the bijection $\phi$ in Section 3, we obtain characterizations of permutation tableaux corresponding to noncrossing linked partitions and nonnesting linked partitions. To be more specific, we have the following correspondences.

Theorem 4.1. For $n \geqslant 1$, there is a bijection between noncrossing linked partitions of $[n]$ and $J_{2}$-avoiding permutation tableaux of length $n$, and there is a bijection between nonnesting linked partitions of $[n]$ and $I_{2}$-avoiding permutation tableaux of length $n$.

Since the number of noncrossing linked partitions of $[n]$ equals the number of nonnesting linked partitions of $[n]$, see Chen, Wu and Yan [1], one sees that the number of $J_{2^{-}}$ avoiding permutation tableaux of length $n$ equals the number of $I_{2}$-avoiding permutation tableaux of length $n$. 


\section{Acknowledgements}

We wish to thank the referee for valuable comments. This work was supported by the 973 Project, the National Science Foundation of China, Beijing Natural Science Foundation, and Beijing Commission of Education.

\section{References}

[1] W.Y.C. Chen, S.Y.J. Wu and C.H. Yan, Linked partitions and linked cycles, European J. Combin. 29 (2008) 1408-1426.

[2] S. Corteel, R. Brak, A. Rechnitzer and J. Essam, A combinatorial derivation of the PASEP stationary state, Electron. J. Combin. 13 (2006) \#R108.

[3] S. Corteel and P. Nadeau, Bijections for permutation tableaux, European J. Combin. 30 (2009) 295-310.

[4] S. Corteel and L.K. Williams, Tableaux combinatorics for the symmetric exclusion process I, Adv. Appl. Math. 37 (2007) 293-310.

[5] S. Corteel and L.K. Williams, A Markov chain on permutations which projects to the PASEP, Int. Math. Res. Not. (2007) Article ID rnm055.

[6] S. Corteel and L.K. Williams, Tableaux combinatorics for the asymmetric exclusion process and Askey-Wilson polynomials, Duke Math. J. 159 (2011) 385-415.

[7] A. de Mier, $k$-Noncrossing and $k$-nonnesting graphs and fillings of ferrers diagrams, Combinatorica 27 (2007) 699-720.

[8] K.J. Dykema, Multilinear function series and transforms in free probability theory, Adv. Math. 208 (2007) 351-407.

[9] The OEIS Foundation Inc., The On-Line Encyclopedia of Integer Sequences, http: //oeis.org.

[10] R.P. Stanley, Enumerative Combinatorics, Vol. I, Cambridge University Press, Cambridge, 1997.

[11] E. Steingrímsson and L. Williams, Permutation tableaux and permutation patterns, J. Combin. Theory Ser. A 114 (2007) 211-234.

[12] X. Viennot, Alternative tableaux, permutations and partially asymmetric exclusion process, Isaac Newton Institute, April 2007. 